\title{
Independent theory versus direct simulation of radiation heat transfer in packed beds
}

\author{
B. P. SINGH and M. KAVIANY \\ Department of Mechanical Engineering and Applied Mechanics, The University of Michigan, \\ Ann Arbor, MI 48109, U.S.A.
}

(Received 3 August 1990 and in final form 14 November 1990)

\begin{abstract}
Radiation heat transfer in packed beds of relatively large spherical particles is considered. The common practice is to follow the theory of independent scattering as long as $C / \lambda>0.5$, where $C$ is the average interparticle clearance and $\lambda$ the wavelength. The single particle properties are related to the radiative properties of the bed by volume averaging. The equation of transfer is then solved by an approximate method such as the method of discrete ordinates or the two-flux method. In this study, the Monte Carlo method is used to examine the thermal radiative transfer through packed beds of large (geometric range) particles. Opaque, semi-transparent and emitting particles are considered. The results are compared to the independent theory and to the available experimental results, and they indicate that the independent theory fails even when this $C / \lambda$ criterion is satisfied. The success of independent theory in systems with low porosities, noted by previous researchers, is shown to be either a special case existing only for a small range of the optical properties or arising due to some unjustifiable assumptions. For the same radiative particle properties, the deviation from the independent theory is shown to increase with decrease in the porosity. This deviation can be significant even for porosities as high as 0.935 .
\end{abstract}

\section{INTRODUCTION}

RADIATIVE heat transfer in packed and fluidized beds can be significant and has attracted considerable interest in the past two decades. The theory of radiative transfer in an absorbing, emitting, and scattering medium is sufficiently developed and can be found in the standard references [1, 2]. The crucial step in applying this theory to packed beds is relating the properties of the packed bed to the properties of an individual particle. To do this, the assumption of independent scattering is introduced, i.e. it is assumed that the interaction of the particle with the radiation field is not influenced by the presence of neighboring particles. This condition is satisfied if the spheres behave as point scatterers, i.e. the distance between two particles is large as compared to their size. Also, there must be no interference between scattered fields. The first condition should lead to a limit on the porosity while the second condition would limit the minimum value of $C / \lambda$, where $C$ is the average interparticle spacing based on a rhombohedral packing $(C / d=$ $\left.0.9047 /(1-\varepsilon)^{1 / 3}-1\right)$. If both these conditions are satisfied, then the bulk (away from the bounding surfaces) behavior of the bed can be predicted from the equation of radiative transfer, by the theory of independent scattering.

The limits of the theory of independent scattering have been experimentally investigated by Hottel $e t a l$. [3]. They identified the limits of independent scattering as $C / \lambda>0.4$ and $C / d>0.4$ (i.e. $\varepsilon>0.73$ ). For a $5 \%$ deviation from the independent theory, they recommend $C / \lambda>0.49$. Brewster [4] also considered larger particles (maximum value of $\alpha_{r}=74$ ). His results indicated that no dependent effects occur as long as $C / \lambda>0.3$, even for a close pack arrangement $(\varepsilon=0.3)$. It was suggested by Brewster [4] that the point scattering assumption is the only artifice necessary in the derivation of the theory and is not crucial to its application or validity. Thereafter, the $C / \lambda$ criterion for the applicability of the theory of independent scattering was verified by Yamada et al. [5] $(C / \lambda>0.5$ for $5 \%$ deviation from the independent theory) and by Drolen and Tien [6]. However, Ishimaru and Kuga [7] noted dependent effects at much higher values of $C / \lambda$. In sum, the above experiments seem to have developed confidence in application of the theory of independent scattering in packed beds consisting of large particles, where $C / \lambda$ almost always has a value much larger than the above-mentioned limit of the theory of independent scattering. Thus the approach of obtaining the radiative properties of the packed beds from the independent properties of an individual particle has been applied to packed beds without any regard to their porosity $[4,6]$. However, all the above experiments were similar in design and most of these experiments used suspensions of small, transparent, latex particles. Only in the experiment of Brewster was a close packing of large, semi-transparent spheres considered.

Dependent scattering involves two distinct effects. The first is the far ficld interference between the scattered waves, which has been studied by Cartigny $e t$ al. [8]. They indicated no observable dependent scattering effects for $\alpha_{\mathrm{r}}>10$. The far field interference affects only the scattering characteristics of the medium and follows the $C / \lambda$ criterion. The second is the effect of multiple scattering in a representative 


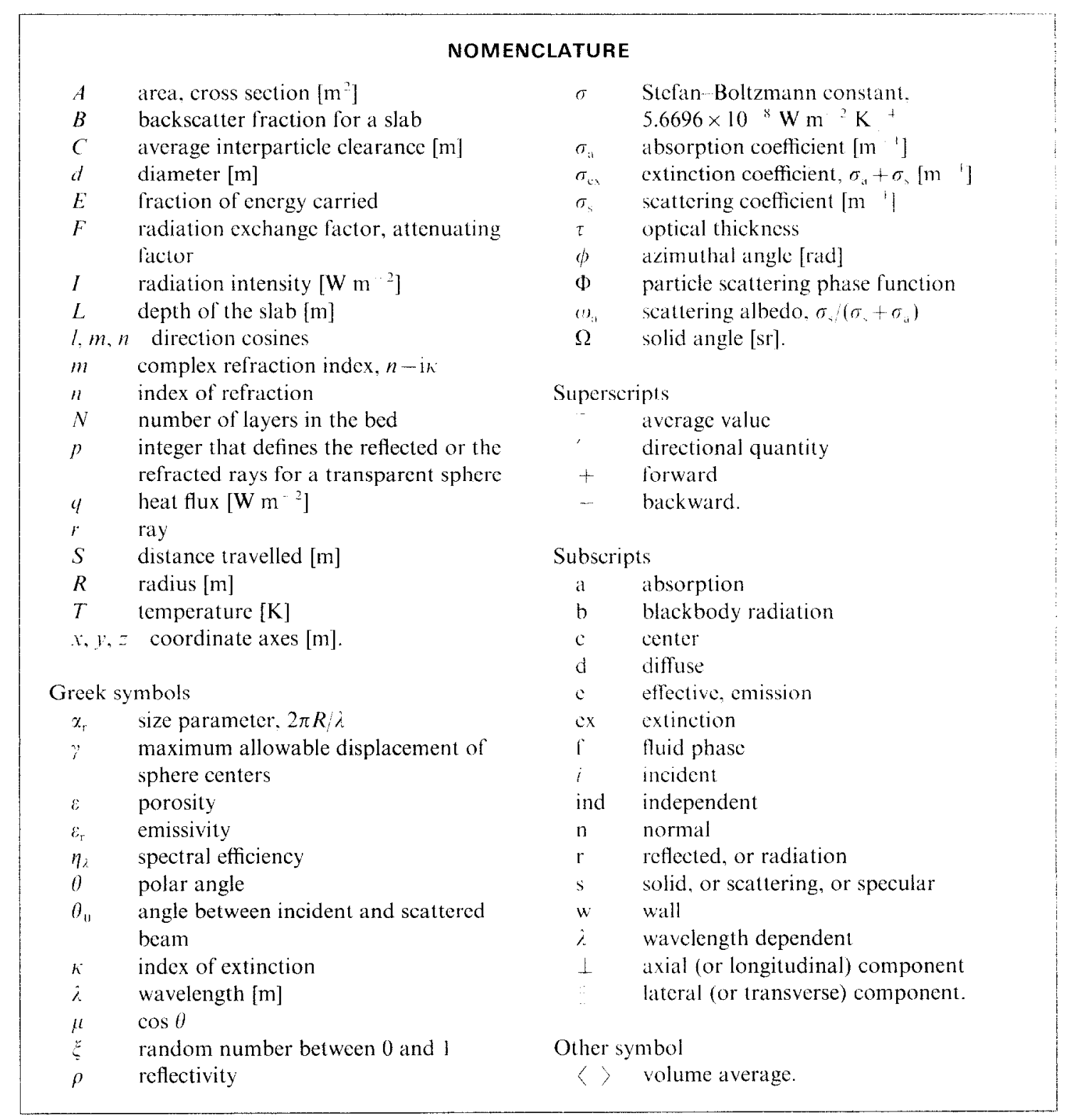

elementary volume in which the scattering and absorption characteristics of the particle are affected by the proximity of other particles. This was studied for small (Rayleigh) sized particles by Kumar and Tien [9].

Other researchers have used the Monte Carlo method to predict the radiative heat transfer in packed beds. Yang et al. [10] studied radiative transfer through a bed of randomly packed, specularly scattering spheres. Kudo et al. [11] considered diffusely scattering particles. Tien and Drolen [12] compared the predictions from the various models with existing experimental results [13] and concluded that the independent theory gives a better prediction than the Monte Carlo method. The Monte Carlo method has also been criticized because it was believed that it could not treat semi-transparent particles.
The case of absorbing and emitting particles is also reviewed by Tien and Drolen [12] and by Vortmeyer [14]. However, the theory of independent scattering fails to satisfactorily explain the experimental results for this very important case. Most notably, the independent theory cannot explain the effect of particle emissivity on the radiative heat transfer. Further discussion of radiative heat transfer in porous media can be found in ref. [15].

In the present study, radiative transfer in packed beds of large (geometric range) size particles is studied using the Monte Carlo method. The far field interference effects, which follow the $C / \lambda$ criterion. are negligible because particles with very large $d$ will almost always have a high value of $C$. The Monte Carlo technique has been extended to accommodatc emitting particles as well as semi-transparent partieles. 
Also, the Monte Carlo simulations over a range of porosities are compared to the results obtained from the equation of radiative transfer and the deviation from the independent theory is shown for smaller porosities. Thus, dependent scattering and absorption are shown to exist even for infinitely large particles, which should fall into the independent range according to the $C / \lambda$ criterion.

\section{CONTINUUM TREATMENT}

The one-dimensional, steady-state, equation of radiative transfer for an absorbing, emitting, and scattering continuum is [1]

$$
\begin{aligned}
\frac{\partial I_{\lambda}}{\partial S}=\left\langle\sigma_{\lambda \mathrm{a}}\right\rangle & I_{\lambda \mathrm{b}}(S)-\left(\left\langle\sigma_{\lambda \mathrm{a}}\right\rangle+\left\langle\sigma_{\lambda s}\right\rangle\right) I_{\lambda}(S) \\
+ & \frac{\left\langle\sigma_{\lambda \mathrm{s}}\right\rangle}{2} \int_{-1}^{1} I_{\lambda}\left(S, \mu_{i}\right)\left\langle\Phi_{\lambda}\right\rangle\left(\mu_{i}, \mu\right) \mathrm{d} \mu_{i}
\end{aligned}
$$

where $I_{\lambda}$ is the spectral intensity, $S$ the distance travelled, $\sigma_{i \mathrm{a}}$ and $\sigma_{\lambda_{s}}$ the spectral absorbing and scattering coefficients, $I_{\lambda \mathrm{b}}$ the blackbody emission, and $\Phi_{\lambda}\left(\mu_{i}, \mu\right)$ the phase function for scattering from a direction $\mu_{i}$ to a direction $\mu(\mu=\cos \theta)$.

The above equation can be written for a packed bed of particles. The scattering and absorption coefficients and the scattering phase function of the medium can either be calculated assuming independent scattering or by modelling the dependent effects. This is discussed in detail in the next section.

\subsection{Calculation of radiative properties}

The calculation of the radiative properties may be done under the assumption of dependent or independent scattering. Independent scattering is rather easy to implement as the radiative properties of a single particle can readily be obtained from the Mie theory or a simpler asymptotic method, e.g. the geometric scattering or the Rayleigh scattering. A model of dependent scattering must consider an assembly of particles. At present, short of a Monte Carlo simulation, no satisfactory model of the dependent scattering for large particles is available. However, according to the currently accepted criterion [12], packed beds of large (geometric) sized particles almost always lie in the regime of the independent scattering. Thus, for packed beds, the theory of independent scattering is generally followed and the radiative properties of the bed are calculated from the properties of the individual particles.

2.1.1. Properties of an individual particle. The spectral scattering efficiency $\eta_{\lambda s}$ is defined as

$$
\eta_{\lambda \mathrm{s}}=\frac{\int_{4 \pi} I_{\lambda \mathrm{s}} r^{2} \mathrm{~d} \Omega}{\pi R^{2} I_{\lambda i}} .
$$

The spectral scattering cross section is defined as

$$
A_{\text {גs }}=\eta_{\lambda s} \pi R^{2}
$$

similarly the spectral absorption efficiency and cross section are defined as

$$
\begin{gathered}
\eta_{\lambda \mathrm{a}}=\frac{\int_{4 \pi} I_{\lambda \mathrm{a}} r^{2} \mathrm{~d} \Omega}{\pi R^{2} I_{\lambda i}} \\
A_{\lambda \mathrm{a}}=\eta_{\lambda \mathrm{a}} \pi R^{2} .
\end{gathered}
$$

Finally the extinction efficiency and cross section are defined as

$$
\begin{gathered}
\eta_{\lambda \mathrm{ex}}=\eta_{\lambda \mathrm{s}}+\eta_{\lambda \mathrm{a}} \\
A_{\lambda \mathrm{ex}}=A_{\lambda \mathrm{s}}+A_{\lambda \mathrm{\lambda a}} .
\end{gathered}
$$

For large particles $\left(\alpha_{\tau}>100\right)$ the diffraction is focused in a highly forward direction and can thus be neglected. Then, the extinction cross section is equal to the geometrical cross section, i.e. the extinction efficiency is equal to one. The scattering efficiency is equal to the hemispherical reflectivity of the sphere [1].

The phase function for specular scatterers with constant reflectivity is isotropic. For diffuse scattering, the phase function is given by [1]

$$
\Phi\left(\theta_{0}\right)=\frac{8}{3 \pi}\left(\sin \theta_{0}-\theta_{0} \cos \theta_{0}\right)
$$

where

$$
\cos \theta_{0}=\cos \theta \cos \theta_{i}+\sin \theta \sin \theta_{i} \cos \left(\phi-\phi_{i}\right) .
$$

For transparent particles, the phase function for an individual particle can be obtained from the theory of geometric optics, by ray tracing, and can be expressed in a series of Legendre polynomials.

2.1.2. Properties of the packed bed. In obtaining the properties of a packed bed, the independent theory assumes the following:

- No interference between the scattered waves (far field effects). This leads to a limit on the minimum value of $C / \lambda$. However, most packed beds are made up of large particles and can therefore be assumed to be above any such limit.

- Point scattering, i.e. the distance between the particles is large compared to their size. Thus a representative elementary volume containing many particles can be found in which there is no multiple scattering, and each particle scatters as if it were alone. Then, this small volume can be treated as a single scattering volume.

- The variation of intensity across this elemental volume is not large.

Then, the radiative properties of the particles can be averaged across this small volume by adding their scattering (absorbing) cross sections. The total scattering (absorbing) cross sections divided by this volume gives the scattering (absorbing) coefficient. The 
phase function of the single scattering volume is the same as that for a single particle.

Using the number of the scatterers per unit volume $N_{s}$ (particles $\mathrm{m}^{\cdot 3}$ ) and assuming independent scattering from each scatterer, the spectral scattering coefficient for uniformly distributed. mono-size scatterers is defined as

$$
\left\langle\sigma_{i s}\right\rangle=N_{s} A_{i s} .
$$

Similarly $\left\langle\sigma_{\text {,a }}\right\rangle=N_{\mathrm{s}} A_{\text {,al }}$, and we also have $\left\langle\sigma_{\text {, ck }}\right\rangle=$ $\left\langle\sigma_{\text {is }}\right\rangle+\left\langle\sigma_{\text {ia }}\right\rangle$.

The optical thickness of an absorbing and scattering medium is defined as

$$
\tau=\int_{0}^{x}\left(\sigma_{\mathrm{s}}+\sigma_{\mathrm{a}}\right) \mathrm{d} x^{*} .
$$

For a medium of mono-size, large particles $\left(\eta_{\mathrm{ex}}=1\right)$, the above equation can be simplified to

$$
\tau=1.5(1-\varepsilon)_{d}^{x}
$$

where $\varepsilon$ is the porosity of the medium.

It is obvious that the condition of point scattering does not, in theory, exist in packed beds. Therefore, as pointed out by Brewster [4], the application of the independent theory to packed beds is only justified by its successful prediction of experimental results (even in systems where it is theoretically not valid).

\subsection{Methods of solution}

2.2.1. Two-flux method. The two-flux approximation involves the assumption that the intensity at any point can be divided into a forward $I_{i}^{+}$and a backward $I_{;}^{-}$component. Then, assuming no emission, the equation of radiative transfer (equation (1)) can be integrated over the forward and the backward directions to give

$$
\begin{aligned}
\frac{\mathrm{d} I_{i}^{+}}{\mathrm{d} x} & =-\left(\bar{\sigma}_{i s}+\bar{\sigma}_{\lambda \mathrm{a}}\right) I_{\lambda}^{+}+\bar{\sigma}_{i s} I_{\lambda}^{-} \\
-\frac{\mathrm{d} I_{i}}{\mathrm{~d} x} & =-\left(\bar{\sigma}_{i s}+\bar{\sigma}_{\lambda \mathrm{a}}\right) I_{\lambda}^{-}+\bar{\sigma}_{i s} I_{\lambda}^{+}
\end{aligned}
$$

where

$$
\begin{gathered}
B=\frac{1}{2} \int_{0}^{1} \int_{-1}^{0}\left\langle\Phi_{i}\right\rangle\left(\theta_{i} \rightarrow \theta\right) \mathrm{d} \cos \theta_{i} \mathrm{~d} \cos \theta \\
\bar{\sigma}_{\lambda s}=2\left\langle\sigma_{i s}\right\rangle B, \bar{\sigma}_{\lambda \mathrm{a}}=2\left\langle\sigma_{\lambda \mathrm{i}\rangle}\right\rangle, \text { and } \\
\left\langle\Phi_{\lambda}\right\rangle\left(\theta_{i} \rightarrow \theta\right)=\frac{1}{\pi} \int_{0}^{\pi}\left\langle\Phi_{i}\right\rangle\left(\theta_{0}\left(\theta_{i}, \phi_{i} \rightarrow \theta, \phi\right)\right) \mathrm{d}\left(\phi-\phi_{i}\right) .
\end{gathered}
$$

For transmission through a bed subjected to incident radiation at one face, the boundary conditions are

$$
I_{\dot{\lambda}}^{+}=I_{i, i} \quad \text { at } \quad x=0
$$

$I_{j}^{-}=0 \quad$ (transparent boundary) at $\quad x=L$.

The solution for the transmittance $T_{\mathrm{r}}$ is

$$
\begin{aligned}
& T_{r}{ }^{1}=\frac{I_{\lambda i}}{I_{\lambda}}=\cosh \left[\left(\bar{\sigma}_{\lambda \mathrm{a}}^{2}+2 \bar{\sigma}_{\lambda \mathrm{a}} \bar{\sigma}_{\lambda \mathrm{s}}\right)^{1 / 2} L\right] \\
& \quad+\frac{\bar{\sigma}_{\lambda \mathrm{s}}+\bar{\sigma}_{\lambda \mathrm{a}}}{\left(\bar{\sigma}_{\lambda \mathrm{a}}^{2}+2 \bar{\sigma}_{j \mathrm{a}} \bar{\sigma}_{\lambda \mathrm{s}}\right)^{1 / 2} \sinh \left[\left(\bar{\sigma}_{\dot{\lambda l} \mathrm{a}}^{2}+2 \bar{\sigma}_{\lambda \mathrm{a}} \bar{\sigma}_{\lambda \mathrm{s}}\right)^{1 / 2} L\right]}
\end{aligned}
$$

and the reflectance $R_{\mathrm{r}}$ is given by

$$
R_{\mathrm{r}}=T_{\mathrm{r}} \frac{\bar{\sigma}_{\lambda \mathrm{s}}}{\left(\bar{\sigma}_{\lambda \mathrm{il}}^{2}+2 \bar{\sigma}_{\lambda . s} \bar{\sigma}_{\lambda \mathrm{a}}\right)^{1 / 2}} \sinh \left[\left(\bar{\sigma}_{\lambda \mathrm{it}}^{2}+2 \bar{\sigma}_{\lambda \mathrm{a}} \bar{\sigma}_{\dot{\lambda} \mathrm{s}}\right)^{1: 2} L\right] .
$$

The fundamental assumption upon which the two flux model is based is that of hemispherical isotropy. Therefore, for the two-flux model to give acceptable results, the scattering must be quasi-isotropic, and the boundary conditions must be diffuse. However, in the case of a non-emitting (in the wavelength range of interest) bed, the hemispherical isotropy is destroyed by the presence of absorption. The error caused by the two-flux assumption increases with increasing absorption and can be significant (as will be shown in Section 5.1).

2.2.2. Method of discrete ordinates. The method of discrete ordinates is an extension of the two-flux method in which the number of discrete streams is increased from 2 to $2 N$. The result of the approximation is to reduce the integro-differential equation to a set of coupled, ordinary, linear differential equations, that are solved numerically (e.g. Carlson and Lathrop [16], Fiveland [17], and Kumar et al: [18]). The in-scattering (integral) term is approximated by a quadrature, where $\mu_{i}$ is the quadrature points between -1 and 1 corresponding to a $2 N$-order quadrature. and $\Delta \mu_{i}$ (solid angle increment) is the corresponding quadrature weight. Then, the one-dimensional radiative transfer equation for intensity at $x$ and in the direction $\mu_{i}$ becomes

$$
\begin{aligned}
& \mu_{i} \frac{\mathrm{d} I_{\lambda j}(x)}{\mathrm{d} x}=-\left\langle\sigma_{\lambda \mathrm{cx}}\right\rangle I_{\lambda, \lambda}(x)+\left\langle\sigma_{i \mathrm{it}}\right\rangle I_{\lambda \mathrm{b}}(x) \\
& +\frac{\left\langle\sigma_{i \mathrm{i}}\right\rangle}{2} \sum_{j=-M_{. j} \neq 0}^{M} \Delta \mu_{j} I_{i j}(x)\left\langle\Phi_{j}\right\rangle\left(\mu_{i} \rightarrow \mu_{j}\right) \\
& \text { for } i=-M,-M+1, \ldots, M, i \neq 0 \\
& \sum_{i, M_{j}+0}^{M} \Delta \mu_{i}=2
\end{aligned}
$$

where

$$
\begin{aligned}
& \left\langle\Phi_{\lambda}\right\rangle\left(\mu_{i}, \mu_{j}\right) \\
& \quad=\frac{1}{\pi} \int_{0}^{\pi}\left\langle\Phi_{j}\right\rangle\left(\theta_{0}\left(\mu_{i}, \phi_{i} \rightarrow \mu_{j}, \phi_{j}\right)\right) \mathrm{d}\left(\phi_{j}-\phi_{i}\right) .
\end{aligned}
$$

The boundary conditions are

at $x=0$

$I_{\lambda i, i}=\varepsilon_{j} I_{\lambda \mathrm{b}}+\rho_{\lambda i s} I_{\lambda \ldots i}+2 \rho_{i \mathrm{~d}} \sum_{j=\cdots 1}^{M} \Delta \mu_{j} I_{\lambda,} \mu_{i}$

$$
i=1, \ldots, M
$$


at $x=L$

$$
\begin{aligned}
I_{\lambda i}=\varepsilon_{\lambda} I_{\lambda \mathrm{b}}+\rho_{\lambda \mathrm{s}} I_{\lambda-i}+2 \rho_{\lambda \mathrm{d}} \sum_{j=1}^{M} \Delta \mu_{j} I_{\lambda j} \mu_{j} \\
i=-1, \ldots,-M
\end{aligned}
$$

where $I_{i}(x)=I\left(x, \mu_{i}\right)$, and $i=0$ (corresponding to the lateral boundaries) has been avoided because of the one-dimensional geometry assumed. In the case of incident radiation on a transparent boundary, the above equation is used with $\varepsilon_{\hat{\lambda}}=1, \rho_{\lambda s}=0$, and $\rho_{\lambda d}=0$. The intensity at the boundary, in a direction $\mu_{i}$, is equal to the intensity of the incident radiation in that direction.

\section{MONTE CARLO SIMULATION}

The ray tracing approach has been used for the study of radiation in packed beds by Chan and Tien [19] who performed ray tracing in a unit cell. Yang et al. [10] applied the Monte Carlo method to a bed of randomly packed specular spheres. They evaluated the absorption and scattcring cocfficients of the packed bed using the solution of the two-flux equations and the results of the Monte Carlo simulations. Kudo et al. [11] considered diffusely reflecting spheres. Both Chan and Tien [19], and Kudo et al. [11] used the unit-cell approach. In extending the results for a unit-cell to a packed bed, they made the assumption of diffuse scattering at each cell face. This is not found to be a valid assumption in the present simulations.

In this study, three different types of particles (opaque, transparent, and emitting) and two types of arrangements are considered. Periodic boundary conditions are applied in the lateral directions to simulate a one-dimensional bed, and the rays are traced through the entire depth of the bed.

\subsection{Arrangements}

Two types of arrangements are considered in the present study. The first is a bed of randomly packed spheres. The bed was generated by the computer program PACKS [20] and has been previously used by Yang et al. [10]. The bed of randomly packed spheres generated by this method has a porosity of 0.42 .

The second is based on a simple cubic packing. The layers however are staggered with respect to each other. This can be significant when considering a packed bed of particles with large absorption because the regular, simple-cubic structure would result in some rays being transmitted directly through the voids. Also, from a practical standpoint, irregular arrangements are more relevant.

The domain of interest consists of a box with a square cross section bounded by $x=0, x=1, z=0$, and $z=1$ and with depth equal to the depth of the bed. The irregular arrangement is achieved by generating sphere centers at four corners of the square $[(0,0)(0,1)(1,0)(1,1)]$ in the $x-z$ planes at $y=0.5$, $1.5, \ldots$. The centers are then staggered by applying the following transformation to all four spheres in the layer:

$$
\begin{aligned}
& x_{\mathrm{c}}=x_{\mathrm{c}}+0.5\left(2 \xi_{x}-1\right) \\
& z_{\mathrm{c}}=z_{\mathrm{c}}+0.5\left(2 \xi_{z}-1\right)
\end{aligned}
$$

where $\xi_{x}$ and $\xi_{z}$ are random numbers between 0 and 1. This process is carried out for each layer using newly generated $\xi_{x}$ and $\xi_{z}$ for each layer. After tracing a small number of rays (say 100 ), the above process is repeated on the original center locations using freshly generated random numbers. Splieres of unit diameter result in a porosity of 0.476 . To get a higher porosity, the sphere size can be reduced. In this case, the spheres will no longer touch each other. For a slower $\varepsilon$, the distance between the layer centers for unit diameter spheres must be $y_{n}-y_{n}{ }_{1}=0.524 /(1-\varepsilon)$, where $y_{n}$ refers to the $y$ coordinate of the $n$th layer. Alternate layers have a sphere at the square center. The layers are staggered by an amount limited by the physical constraint that no overlap is allowed. Thus the maximum distance by which a layer can be of stagger varies from 0.5 for a porosity of 0.476 to 0 for a porosity of 0.26 . The above two equations must now be changed to

$$
\begin{aligned}
& x_{\mathrm{c}}=x_{\mathrm{c}}+\gamma(2 \xi-1) \\
& z_{\mathrm{c}}=z_{\mathrm{c}}+\gamma(2 \xi-1)
\end{aligned}
$$

where $\gamma$ is a function of porosity alone and represents the extent to which sphere centers can be displaced without overlap.

Both of the above models are used in conjunction with the periodic boundary condition in the $x$ - and $z$ directions.

\subsection{Opaque particles}

A ray is defined by the coordinates of its starting point $P_{0}\left(x_{0}, y_{0}, z_{0}\right)$ and its direction cosines $(l, m, n)$. The ray enters the bed at a random point in the $x-z$ plane (forming the lower surface $(y=0)$ ), i.e.

$$
\left(x_{0}, y_{0}, z_{0}\right)=\left(W \xi_{x}, 0, W \xi_{z}\right)
$$

where $W$ is the lateral dimension of the box being used and $\xi_{x}$ and $\xi_{z}$ are random numbers between 0 and 1 . The angles $\phi$ and $\theta$ are given by

$$
\begin{gathered}
\phi=2 \pi \xi \\
\theta=\cos ^{-1}\left(1-\xi\left(1-\cos \theta_{\max }\right)\right)
\end{gathered}
$$

where $\theta_{\max }$ is the maximum angle that the incident radiation makes with the normal. For diffuse incident flux, $\cos \theta_{\max }=0$. The direction cosines of the ray are

$$
\begin{gathered}
l=\sin \theta \cos \phi \\
m=\cos \theta \\
n=\sin \theta \sin \phi .
\end{gathered}
$$


The coordinates of a ray after travelling a length $S$ are given by

$$
\begin{aligned}
& x=x_{10}+l S \\
& y=y_{0}+m S \\
& z=z_{0}+n S .
\end{aligned}
$$

Substituting in the equation of the sphere, we have

$$
\left(x-x_{c}\right)^{2}+\left(y-y_{c}\right)^{2}+\left(z-z_{c}\right)^{2}=R^{2}
$$

i.e. a quadratic equation in $S$ is obtained. A positive discriminant indicates that the ray intersects the sphere. Equation (37) is solved for all spheres for which it has a positive discriminant. The smaller of the two solutions obtained gives the actual point of intersection with a sphere. Also, the distance the ray travels before it intersects a bounding surface is determined. The minimum distance a ray travels before it is intercepted by a sphere or a bounding surface is then determined. The sphere or the bounding surface corresponding to this solution is the surface which actually intercepts the ray.

If the ray is intercepted by the side walls, the periodic boundary condition is applied. In case it passes through the upper or the lower face, the energy associated with the ray is registered as transmission or reflection. If it is intercepted by a sphere, the point of intersection is determined and the direction cosines of the reflected ray for a specularly scattering sphere are found using the laws of reflection, i.e.

- The incident ray, the reflected ray and the normal to the surface all lie in the same plane.

- The angle of incidence is equal to the angle of reflection.

If the sphere is assumed to be diffusely scattering, then the ray is scattered in a random direction from the point of interception under the restriction that the ray does not penetrate the sphere. After reflection, the energy of the reflected ray is given by $E_{\mathrm{r}}=\rho E_{i}$. A combination of diffuse and specular scattering can also be modelled. This can be done by generating a random number at every collision and comparing it to the ratio of specular scattering to the total scattering. Then, the scattering is allowed to take place specularly if the random number is less than the fraction scattered specularly. Otherwise, the ray is scattered diffusely.

The above process is repeated until the ray passes through either the upper or the lower surface. The number of rays used for each simulation ranged from 100000 to 1000000 . Packed beds with lower transmittance need more rays for the same accuracy.

\subsection{Semi-transparent particles}

Transmitting particles are dealt with by ray tracing inside the sphere, following the laws of reflection and refraction. The angle that the incident radiation makes with the tangent to the surface, i.e. $\left(\theta_{i}\right)$, is calculated. Then, the angle of refraction is given by

$$
\cos \theta_{i}=n \cos \theta_{\mathrm{r}}, \quad n=\frac{n_{i}}{n_{i}} .
$$

Next, the Fresnel coefficients and the reflectivity are calculated in terms of the angles $\theta_{i}$ and $\theta_{\mathrm{r}}$ (pp. 95101 of Siegel and Howell [1]), i.e.

$$
\begin{aligned}
& \rho_{i j}^{\prime}=\left[\frac{\tan \left(\theta_{i}-\theta_{\mathrm{r}}\right)}{\tan \left(\theta_{i}+\theta_{\mathrm{r}}\right)}\right]^{2} \quad \text { and } \\
& \rho_{i j}^{\prime}=\left[\begin{array}{l}
\sin \left(\theta_{i}-\theta_{\mathrm{r}}\right) \\
\sin \left(\theta_{i}+\theta_{\mathrm{r}}\right)
\end{array}\right]^{2} \text { for } k \rightarrow 0 .
\end{aligned}
$$

Thus, the reflected parts of energy are $\rho_{i ;}^{\prime}$ and $\rho_{1 ;}$. The refracted parts are $1-\rho_{1 ;}^{\prime}$ and $1-\rho_{1}^{\prime}$; Then, the energy carried by the various rays is [21]

$$
\begin{array}{ll}
E_{i, p}=\rho_{i ;}^{\prime} & \text { for } p=0 \\
E_{\mid, p}=\left(1-\rho_{i \lambda}^{\prime}\right)^{2}\left(\rho_{\mid \lambda}^{\prime}\right)^{p-1} & \text { for } p=1,2,3 \ldots \\
& \text { if } \kappa=0 .
\end{array}
$$

For the other polarization, replace $\|$ with $\perp$. However, if $\kappa_{\mathrm{s}}$ is not small, then equation (39) should not be used to calculate the reflectivity. Instead, an exact analysis should be followed (p. 100 of Siegel and Howell [1]) although ray tracing beyond $(p=0)$ will not be required because even moderate values of $\kappa_{\mathrm{s}}$ (for a large particle in the geometrical optics range) make the particle virtually opaque.

For non-polarized irradiation, the total energy carried by a ray is given by

$$
E_{p}=\frac{1}{2}\left(E_{\mathrm{L}^{\prime},}+E_{\mathrm{t}, p}\right) .
$$

When a ray strikes a sphere, it is either reflected $(p=0)$ or transmitted $(p=1,2 \ldots)$ with a reduction in the energy due to absorption. The outcome is decided by generating a random number. Let us define

$$
\beta_{i}=\sum_{j-0}^{1} E_{j} .
$$

Then, the ray is reflected $(p=0)$ if

$$
\check{\zeta}<\beta_{0}
$$

and is transmitted, with $p=i$, if

$$
\beta_{i}<\xi \leqslant \beta_{i+1} .
$$

Generally, tracing up to $p=2$ or 3 is sufficient. Figure 1 shows a sketch of a ray traced up to $p=2$.

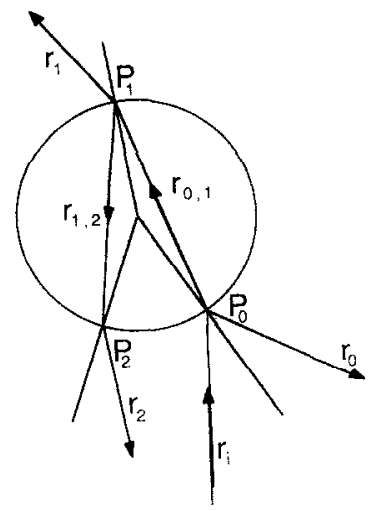

FIG. I. Ray tracing for a semi-transparent sphere 
The ray incident on the point $P_{0}$ can either be reflected or transmitted.

- If the ray is reflected, its direction cosines are calculated as in the case of opaque particles. However, the energy carried by the ray, remains unchanged.

- If the ray is transmitted, the direction cosines of the ray $r_{0,1}$ are found using the laws of refraction.

- the incident ray, the refracted ray, and the normal to the surface all lie in the same plane;

- the angle of refraction is related to the angle of incidence by Snell's law (equation (38)).

The coordinates of point $P_{1}$ are found by using $P_{0} P_{1}=2 R \sin \theta_{\mathrm{r}}$. The direction cosines of the rays $r_{1,2}$ and $r_{1}$ are found by applying the laws of reflection and refraction at point $P_{1}$, respectively.

The coordinates of point $P_{2}$ and the direction cosines of ray $r_{2}$ are found by repeating the above steps.

In the case of semi-transparent particles $(\kappa \neq 0)$, the energy of the rays is reduced by an attenuating factor given by

$$
F_{p}=\exp \left(-4 p \kappa \alpha_{\mathrm{r}} \sin \theta_{\mathrm{r}}\right) \text { for } p=1,2,3, \ldots
$$

where $\alpha_{\mathrm{r}}$ is the size parameter $\left(\alpha_{\mathrm{f}}=2 \pi R / \lambda\right)$. Therefore, the energy carried by a transmitted ray is given by

$$
E_{\mathrm{r} p}=F_{p} E_{i} \quad \text { for } p=1,2, \ldots
$$

\subsection{Emitting particles}

The spheres are assumed to have a high enough thermal conductivity so that a sphere can be assumed to be isothermal. The case simulated here is of a bed of absorbing, emitting, and scattering spheres. If the sphere has a reflectivity $\rho$, the ray is :

- Reflected if $\rho>\xi$; either diffuse or specularly reflecting particles may be considered.

- Absorbed and emitted if $\rho \leqslant \xi$.

The emission can take place from any randomly selected point on the surface. Also, the direction of the emitted ray is determined according to the Lambert cosine law as in the case of diffusely reflected rays.

\section{RADIANT CONDUCTIVITY}

The radiative heat transfer for a one-dimensional, plane geometry, with emitting particles under steadystate condition is given by [14]

$$
q_{\mathrm{r}}=\frac{F \sigma}{\frac{1+\rho_{\mathrm{w}}}{1-\rho_{\mathrm{w}}}=\frac{L}{d}}\left(T_{1}^{4}-T_{2}^{4}\right)
$$

where $F$ is called the exchange factor and the properties are assumed to be wavelength independent. If $\rho_{\mathrm{w}}=\mathbf{0}$ and the bed is several particles deep, then the first term of the denominator can be neglected. Then, for $T_{1}-T_{2}<200 \mathrm{~K}$, a radiant conductivity is defined [12]

$$
k_{\mathrm{r}}=4 F d \sigma T_{\mathrm{m}}^{3} .
$$

Many different models are available for prediction of $F$, and these are reviewed by Vortmeyer [14]. Here, the main emphasis will be on examining the validity of this approach, by comparing the results of some of these models with the Monte Carlo simulations and also with the available experimental results.

A solution to this problem based on the two-flux model is given by Tien and Drolen [12]

$$
F=\frac{2}{d\left(\bar{\sigma}_{\lambda \mathrm{a}}+2 \bar{\sigma}_{\lambda \mathrm{s}}\right)}
$$

which can be written as

$$
F=\frac{2}{3 f_{\mathrm{v}}\left(\eta_{\lambda \mathrm{a}}+2 B \eta_{\lambda \mathrm{s}}\right)} .
$$

For isotropic scaltering, $B=0.5$ and the above equation becomes independent of the particle emissivity.

\section{RESULTS AND DISCUSSION}

\subsection{Examination of two-flux method}

The inability of the two-flux model to handle large anisotropy in the phase function was noted by Brewster and Tien [22] and by Mengüc and Viskanta [23] although it was found to be suitable for isotropic scatterers. However, only non-absorbing media were considered in these studies $\left(\omega_{a}=1\right)$. It is shown here that the two-flux model fails to handle the hemispherical anisotropy created by an isotropically scattering, absorbing medium. We consider a packed bed of specularly reflecting spheres with constant reflectivity of 0.6 and porosity of 0.40 subject to a diffuse source on one of its boundaries. Then, we calculate the properties of the medium as described in Section 2.2 and solve the equation of radiative transfer by applying the two-flux method and the discrete ordinates method (using a 24 point Gaussian quadrature). The results are presented in Fig. 2. The figure clearly

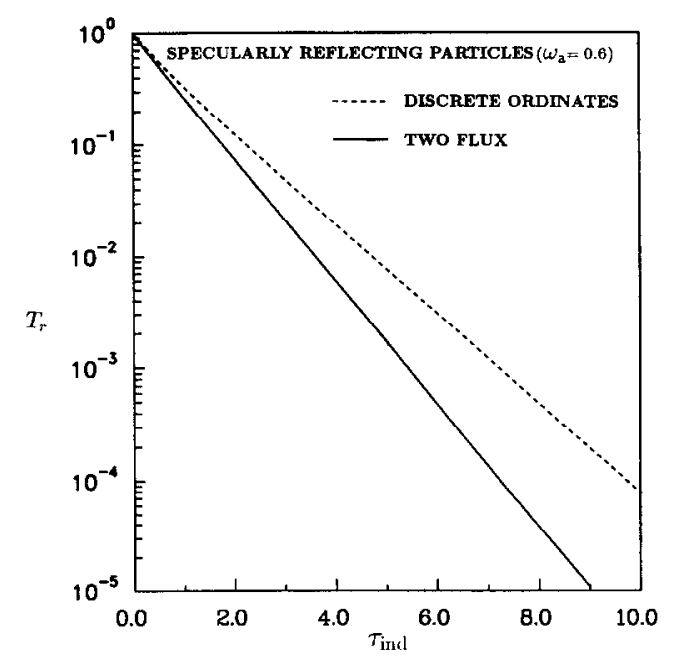

FIG. 2. Effect of optical thickness on transmittance for an absorbing medium $\left(\omega_{\mathrm{a}}=0.6\right)$. 
shows that the two-flux model fails to give satisfactory results.

The above point could have been made for an ideal medium with given properties $\left(\left\langle\sigma_{\mathrm{a}}\right\rangle,\left\langle\sigma_{\mathrm{s}}\right\rangle\right.$, and $\left.\langle\Phi\rangle\right)$ without refering to a packed bed. However, the abovementioned set of conditions resembles the experiment of Chen and Churchill [13], where the use of the twoflux method is partly responsible for incorrect conclusions regarding the validity of the theory of independent scattering in packed beds. This will be discussed in detail in Section 5.3.

\subsection{Comparison of Monte Carlo simulations and independent theory}

The Monte Carlo method, being a direct simulation, involves very few assumptions. On the other hand, the continuum treatment involves the approximation of a heterogeneous system by a single continuum. The major task is that of obtaining the radiative properties of this continuum. This can be done by using the independent theory (which though easiest to use, is not always valid), or through alternate methods that allow for interparticle interactions. The Monte Carlo method can be used to get the transmittance or reflectance through a packed bed (as is done in this section). Alternatively, the radiative properties of the bed can also be obtained using the Monte Carlo method. These properties are not uniform since the bulk behavior and that near bounding surfaces are different.

According to the currently accepted criterion, packed beds of large particles lie in the independent regime; therefore, the independent theory should be valid for these beds. However, it will be shown in this section that because of the violation of the point scattering assumption, the theory of independent scattering breaks down when the porosity is small. As the porosity is increased, a closer agreement is expected between the Monte Carlo simulation and the results obtained from the equation of transfer solved by the method of discrete ordinates. In this section, first opaque particles, and then semi-transparent particles are examined. The particles are assumed to be nonemitting in the wavelength range of interest.

Figure 3 shows the transmittance through a medium consisting of totally reflecting particles. The scallering is assumed to be specular. Porositics of $0.476,0.732,0.935$, and 0.992 are considered. The bed is generated by the second method described in Section 3.1. The optical thickness is calculated from equation (11). Thus according to the theory of independent scattering, the following systems are exactly equivalent (as far as their radiative behavior is concerned) :

$$
\begin{aligned}
& \bullet \varepsilon=0.476, N=8.0 \\
& \bullet \varepsilon=0.732, N=12.5 \\
& \bullet \varepsilon=0.935, N=32.0 \\
& \bullet \varepsilon=0.992, N=128.0
\end{aligned}
$$

where $N$ is the number of layers in the generated bed

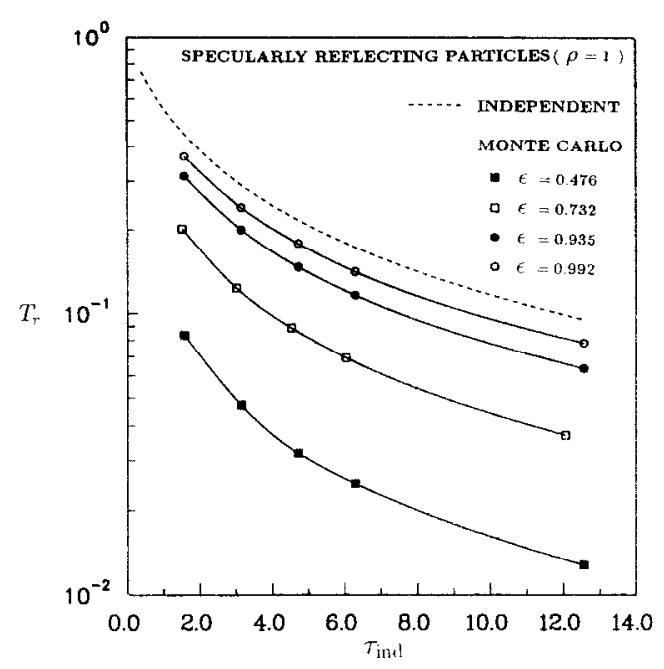

FIG. 3. Effect of porosity on transmittance for totally reflecting spheres $(\rho=1)$.

( $N=L$ for all porosities greater than or equal to the porosity of the simple cubic arrangement since the layers are a unit distance apart). The value of $L / d$ is obtained by noting that for systems having a porosity larger than the simple cubic porosity, $d=$ $((1-8) / 0.524)^{1 / 3}$. Thus, the values of $d$ for the four systems mentioned above are $1.0,0.8,0.5$, and 0.25 , respectively.

All these systems give $\tau_{\text {ind }}=6.28$, where $\tau_{\text {ind }}$ is the optical thickness of a bed based on the independent theory, i.e. the independent theory predicts identical behavior for all these systems. The transmittance through packed beds of different porosities and at different values of $\tau_{\text {ind }}$ was calculated by the method of discrete ordinates using a 24 point Gaussian quadrature. Further increase in the number of ordinates did not cause any significant change in the results. It is clear from Fig. 3 that the independent theory fails for low porosities. As the porosity is increased, the Monte Carlo solution begins to approach the independent theory solution. For $\varepsilon=0.992$, the agreement obtained is good. The bulk (away from the bounding surface) behavior predicted by the Monte Carlo simulations for $\varepsilon=0.992$ and the results of the independent theory are in very close agreement. A small difference occurs at the boundaries, where the bulk properties are no longer valid. However, although this difference occurs at the boundary, the commonly made assumption that the prediction by the continuum treatment will improve with increase in the optical thickness is not justifiable because this off-set is carried over to larger optical thicknesses.

Figure 4 shows the effect of the porosity on transmittance for absorbing particles $(\rho=0.7)$. Again, the independent theory fails for low porosities although the agreement for a dilute system is good. Thus, the transmittance for a packed bed of opaque particles can be significantly less than that predicted by the independent theory. This is due to multiple scattering in a representative elementary volume, so that the 


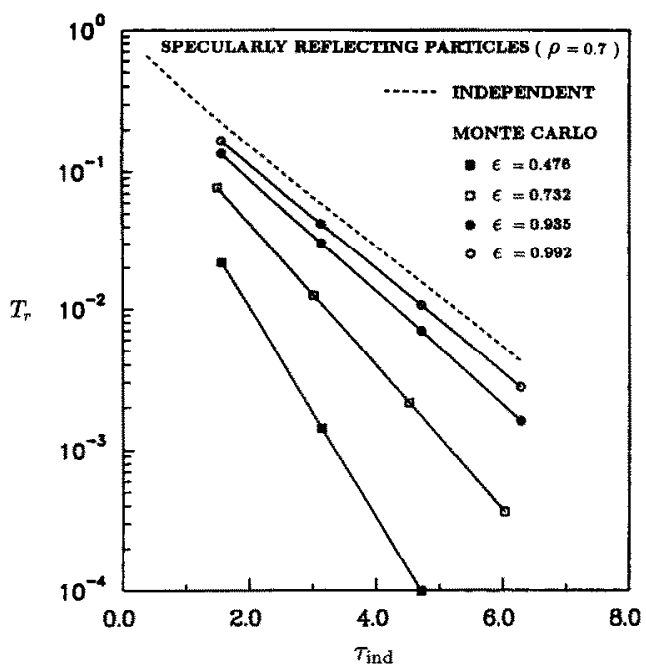

Fig. 4. Effect of porosity on transmittance for absorbing spheres $(\rho=0.7)$.

effective cross section presented by a particle is more than its independent cross section.

Figures 5-7 show the effect of change in the porosity on the transmittance through a medium of semi-transparent particles. The particles considered are large spheres with $n=1.5$. For these particles, the only parameter that determines the radiative properties of a particle is the product $\kappa \alpha_{T}$ (as long as $\kappa$ is not too large). Figure 5 is plotted for the case of $\kappa=0$ (transparent spheres). Differences from opaque particles (Figs. 3 and 4) are obvious. A violation of the independent theory results in a decrease in the transmittance for opaque spheres, but for transparent spheres, it results in an increase in the transmittance. This results because the change in the optical thickness across one particle in a packed bed is large. Therefore, a transparent particle while transmitting the ray through it also 'transports' it across a substantial

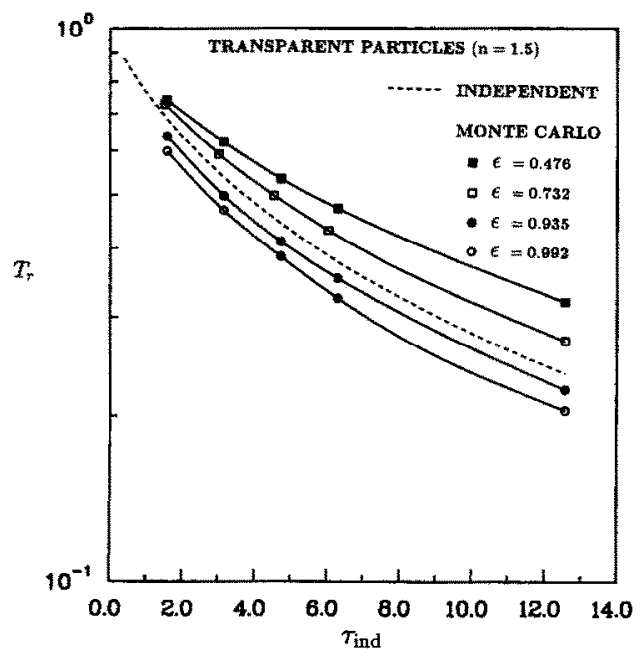

FIG. 5. Effect of porosity on transmittance for transparent spheres $\left(n=1.5, \eta_{\lambda_{a}}=0\right)$.

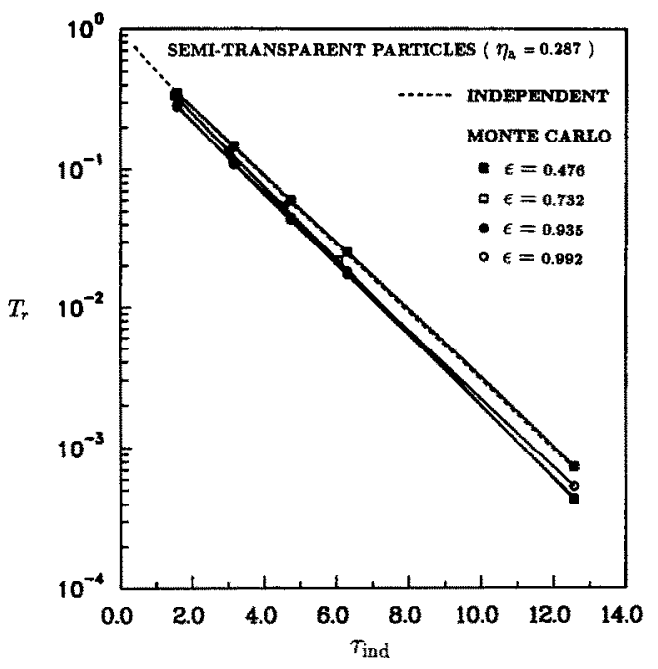

FIG. 6. Effect of porosity on transmittance for semi-transparent spheres $\left(n=1.5, \eta_{\lambda \mathrm{a}}=0.287\right)$.

optical thickness. In a dilute suspension, a particle while transmitting through it still does not transport it across a substantial drop in intensity.

Figure 6 is plotted for semi-transparent particles with $\kappa \alpha_{\mathrm{r}}=0.1$, which gives $\eta_{\lambda \mathrm{a}}=0.287$. The absorption decreases the above effect (transportation across a layer of substantial optical thickness) to the extent that it is exactly balanced by the decrease due to multiple scattering in the elementary volume for $\varepsilon=0.476$. As a result, the Monte Carlo prediction for $\varepsilon=0.476$ shows very good agreement with the prediction from the independent theory. The results for a dilute system are exactly as expected: giving slightly less transmittance than the independent theory solution but showing the same bulk behavior. Therefore, due to these two opposing effects, the magnitude of deviation from independent theory for

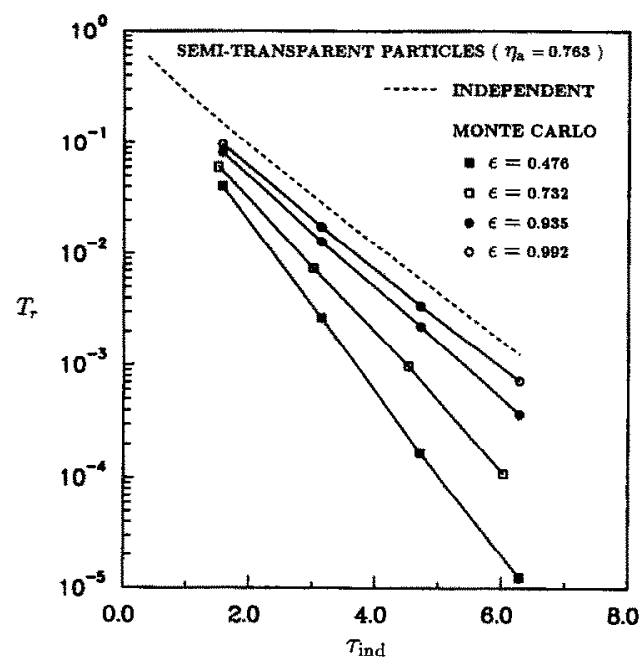

Fig. 7. Effect of porosity on transmittance for semi-transparent spheres $\left(n=1.5, \eta_{\lambda a}=0.763\right)$. 
packed beds of transparent and semi-transparent particles is smaller than that for opaque spheres.

Figure 7 shows the effect of variation in porosity on transmittance through a medium of highly absorbing semi-transparent particles $\left(k \alpha_{\mathrm{r}}=0.5, \eta_{\eta_{\mathrm{a}}}=0.763\right)$. Here, the multiple scattering effect clearly dominates over the 'transportation' effect. The predicted transmittance for low porositics by the Monte Carlo method is far less than that predicted by the independent theory, while the most dilute system $(\varepsilon=0.992)$ again shows good agreement with the independent theory. It is encouraging to note that the $\varepsilon=0.992$ system matched the independent theory results for all cases considered. However, the effect of the porosity on transmittance is noticeable even for relatively high porosities $(\varepsilon=0.935)$.

\subsection{Comparison with the Chen and Churchill exper- iment}

The experiment of Chen and Churchill [13] is the most referenced experiment on radiative transfer in packed beds [12]. In this experiment, an open-ended tubular furnace behaving as a high temperature, blackbody source was incident on one surface of a packed bed of spheres. The flux was modulated to a square wave by a mechanical chopper, and the intensity of the transmitted radiation was measured with a thermopile detector. The packed bed was designed to simulate a one-dimensional bed by using an aluminum tube with highly reflecting walls as the container. The transmission through the bed was measured through isothermal beds of glass, aluminum oxide, steel, and silicon carbide particles of different shapes. The use of the modulator enabled the measurement of only the scattered and directly transmitted radiation from the source and the particles in the bed can be considered to be nonemitting.

Many authors have compared the results of their models to the experimental results of Chen and Churchill. However. one fact which has been overlooked by all of them is the incident boundary condition to be applied. The incident boundary condition has always been treated as diffuse. Figure 8 shows a sketch of the apparatus used in this experiment [24]. It is clear that the incident radiation is collimated from a very small solid angle around the normal. Neither the two-flux model nor the unit-cell type Monte Carlo simulation are capable of handling this boundary condition. However, it can be accommodated by the method of discretc ordinates as well as the direct simulation of a packed bed.

Figure 9 shows the comparison of results obtained from the Monte Carlo method with those from the independent theory (using the method of discrete ordinates). with the Chen and Churchill experiment on steel spheres $(d=4.7625 \mathrm{~mm}, T=1366 \mathrm{~K}$, where $T$ is the temperature of the blackbody source). The experiment was closely matched by Brewster [4] by using an emissivity of 0.4 (as recommended by Chen and Churchill [13]) and by using the two-flux model.

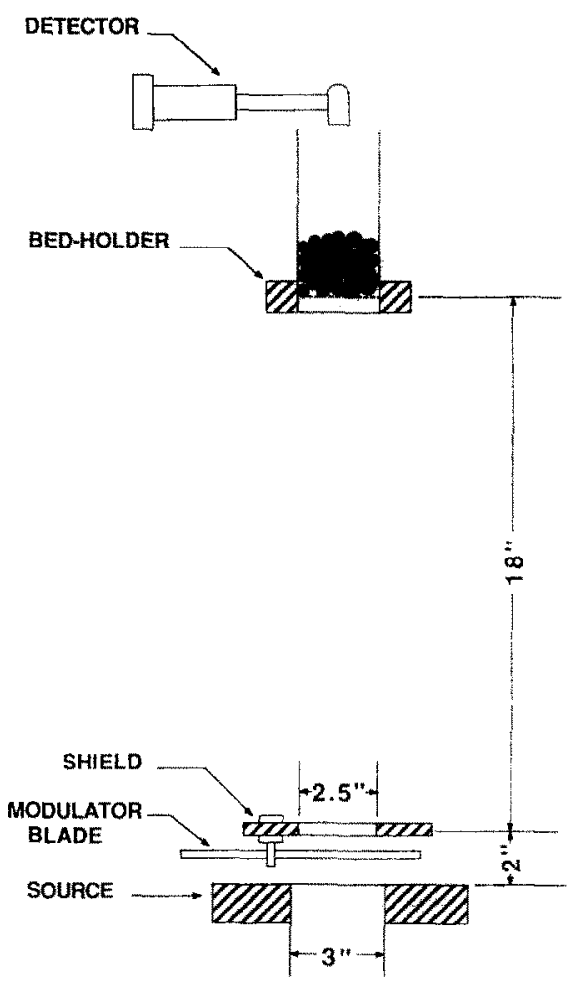

FIG. 8. Relative position of the packed bed and the source in the Chen and Churchill experiment.

The errors resulting from the two-flux method were discussed in Section 5.1. The use of the incorrect boundary condition adds to the error because both of these factors (the use of the two-flux model and the use of the incorrect boundary condition) result in lower transmission. Figure 9 shows the results obtained from the independent theory, assuming specularly reflecting spheres with an emissivity of 0.4 .

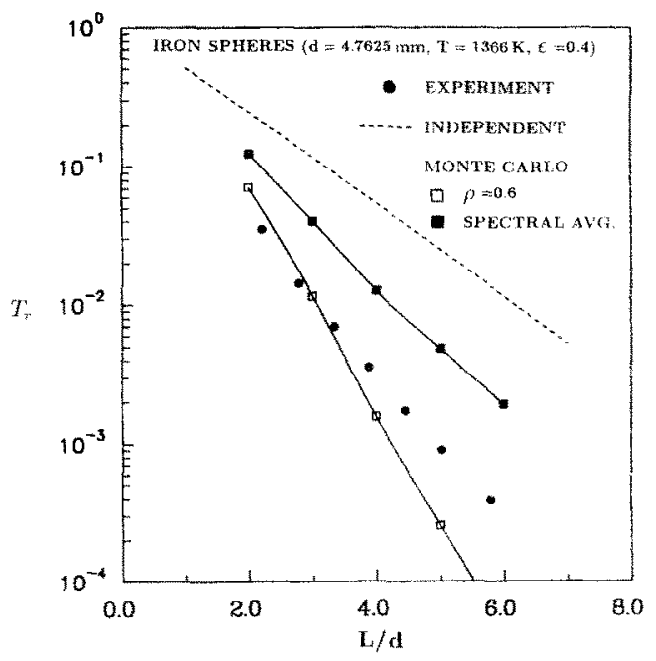

Fic. 9. Transmittance from a bed of steel spheres: comparison of the Chen and Churchill experiment with the theoretical predictions. 
and solving by the method of discrete ordinates. It can be seen that the independent theory predicts a much higher transmittance than the experimental results of Chen and Churchill.

Drolen and Tien [6] calculated the emissivity from the optical properties of iron. They used the $n$ and $\kappa$ values at $\lambda=0.589 \mu \mathrm{m}$ from Siegel and Howell [1] and scaled them to $\lambda=\lambda_{\max }$ (where $\lambda_{\max }$ is the wavelength corresponding to the maximum emissive power in the blackbody spectrum) using the Hagen-Rubens formula. Then using the Mie theory, they arrived at $\eta_{\lambda a}-0.50$. However, the approach of using the optical properties at $\lambda=\lambda_{\max }$ is questionable since $75 \%$ of the energy of the blackbody spectrum is at higher wavelengths. Also, the use of the Hagen-Rubens formula is unnecessary because the optical properties of iron are now available [25]. The spectrum was divided into five wavelength bands, and the wavelengths in the middle of the band were used to calculate the emissivities of a large particle. Emissivity values ranging from 0.05 to 0.30 were obtained. This would result in further worsening of the prediction by the independent theory.

The Monte Carlo method was used with a randomly packed bed $(\varepsilon=0.58)$ generated using the sphere settling program of Jodrey and Tory [20]. Figure 9 shows two plots obtained from the Monte Carlo method. The first is plotted for specularly scattering particles for $\varepsilon_{\mathrm{r}}=0.4$ (as reported by Chen and Churchill). Also plotted is a spectrally averaged transmittance obtained by calculating the transmittance for each band and computing a weighted average. It is clear from the figure that the results from the Monte Carlo method lie in the same range as the experiment. The uncertainty in the emissivity of steel because of the dependence on the temperature and due to the presence of an oxide coating was pointed out by Chen and Churchill [13]. In fact, the first couple of layers might be at a higher temperature than the rest of the bed because of their proximity to the source.

It must be stressed that the independent theory fails to explain the experimental results even when a very large allowance is made for uncertainty in the emissivity.

Figure 10 shows the comparison of results obtained from the Monte Carlo method and the independent theory with the Chen and Churchill experiment for glass spheres $(d=5 \mathrm{~mm}, T=1366 \mathrm{~K})$. The only other prediction available is that of Brewster [4] who used the two-flux method with an emissivity of 0.03 for $\lambda<2.7 \mu \mathrm{m}$, and an emissivity of 0.9 for $\lambda>2.7 \mu \mathrm{m}$. For the present study, the wavelength range was divided into three suitable bands. The optical properties of glass [26] were used to find the absorption efficiencies and the scattering phase function from the theory of geometric scattering. Figure 11 shows the variation of absorption efficiency with wavelength for a $5 \mathrm{~mm}$ glass sphere. The phase was calculated using the theory of geometric scattering and expressed in a series of Legendre polynomials. Then, the method of

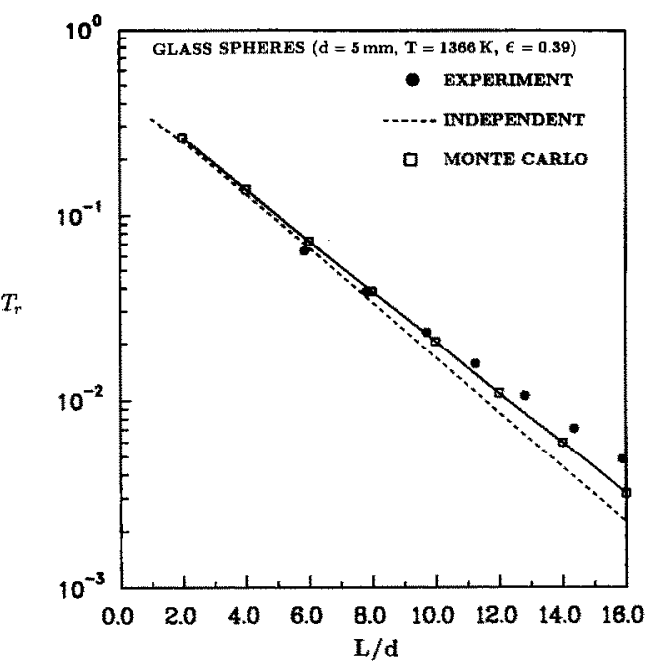

FIG. 10. Transmittance from a bed of glass spheres: comparison of the Chen and Churchill experiment with the theoretical predictions.

discrete ordinates was used to calculate the transmittance predicted by the independent theory. The Monte Carlo simulation for a randomly packed bed of semi-transparent particles was performed. The results for the Monte Carlo simulation show good agreement with the experimental results. The prediction obtained from the independent theory also shows fair agreement. As pointed out in the previous section, this is because the errors resulting from the use of the independent theory, for semi-transparent particles with small absorption, are not very large.

\subsection{Comparison with the Kasparek experiment}

In the Kasparek experiment (described by Vortmeyer [14]) measurements of radiation heat transfer through a number of planar series of welded steel spheres were made. Conduction and convection were

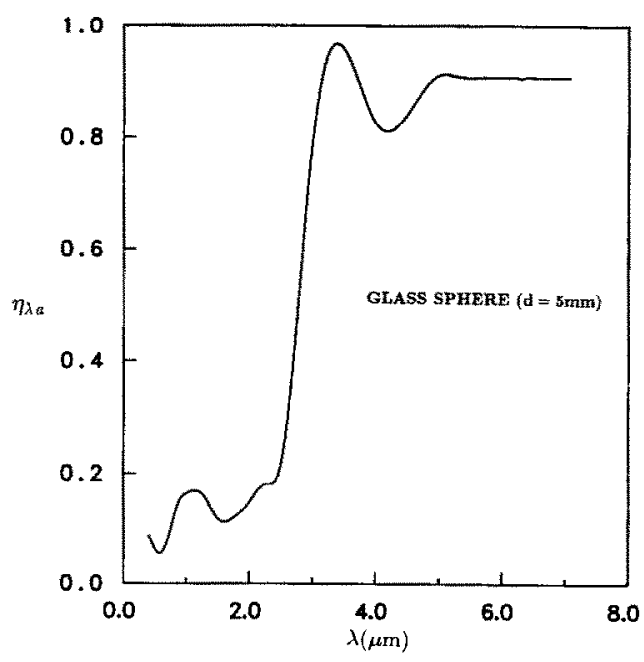

FiG. 11. Absorption efficiency of a glass sphere of diameter $5 \mathrm{~mm}$. 
eliminated by placing the layers a small distance apart and by performing the experiment in vacuum. The high thermal conductivity of the material ensured that the heat resistance of the material was negligible and that the spheres were isothermal. Measurements were made using polished steel spheres $\left(\varepsilon_{\mathrm{r}}=0.35\right)$ and chromium oxide coated spheres $\left(\varepsilon_{\mathrm{r}}=0.85\right)$. The arrangements considered were a cubic $(\varepsilon \approx 0.5)$ and a porosity of 0.4 .

The Monte Carlo method as described in Section 3.3 was used to predict the heat transfer through the packed bed used in the Kasparek experiment (unlike the previous sections, we encounter emitting particles). The results confirm the validity of the exchange factor approach as long as the emissivity is not close to zero. The change in the value of the exchange factor (obtained from equation (48)), resulting from increasing the number of layers from 8 to 16 , was less than 0.01 for $\varepsilon_{\mathrm{r}} \geqslant 0.20$. The simulation was performed for both specularly scattering and diffusely scattering spheres. The polished steel spheres can be considered specularly scattering while the chromium oxide spheres would scatter diffusely. Table I shows the results of the Monte Carlo simulation as well as the results obtained from the two-flux model and those from the models of Argo and Smith (described by Vortmeyer [14]) and Vortmeyer [14] for a porosity of 0.4 . The predictions by the Monte Carlo method match the experimental results fairly well, considering that some uncertainty is always present in the emissivity values. Diffuse spheres result in slightly smaller values of $F$ although, as expected, the difference decreases with increasing emissivity and vanishes for $\varepsilon_{\mathrm{r}}=1$. The change in value of $F$ with porosity also matched the experimental results. For a cubic packing, the value of $F$ increases from 0.47 to 0.51 , for $\varepsilon_{\mathrm{r}}=0.35$, while the experimental value increases from 0.54 to 0.60 . For $\varepsilon_{\mathrm{r}}=0.85$, the value of $F$ increases from 0.94 to 0.97 , while the experimental value increases from 1.02 to 1.06 .

The predictions based on the two-flux model for diffuse spheres show a very small sensitivity to the emissivity, while those for specularly scattering spheres show no change at all. Using the method of discrete ordinates, higher values of $F$ are obtained than those predicted by the two-flux method. Also, for specularly scattering spheres, the heat transfer remained independent of the emissivity. This can be seen from equation (19) by applying the condition of radiative equilibrium. Physically, when the spheres are treated as point scatterers, there is clearly no difference between isotropic scattering from a point and emission from it. The mechanism that results in an increase in the radiative heat transfer, with an increase in the emissivity, is that of transportation of the absorbed energy through each particle (by conduction), i.e. particles absorb radiation at one facc and emit a part of it from the other face. For a dilute medium consisting of isotropically scattering, small particles separated by large distances, the heat transfer is again expected to be independent of the particle emissivity.

\section{CONCLUSIONS}

Radiative heat transfer through a packed bed of particles has been simulated using the Monte Carlo technique, and the results are compared with available experimental results and also the results obtained from the solution of the equation of radiative transfer under the assumption of independent scattering. The important conclusions that can be drawn from this study are:

- The independent theory is shown to fail for systems with low porosity and is not suitable for packed beds (even though the $C / \lambda$ criterion is satisfied). The failure is more drastic for transmission through a bed of opaque spheres than for transparent and semitransparent spheres with low absorption. Also, the deviation from the independent theory is shown to increase with decrease in the porosity. This deviation can be significant for porosities as high as 0.935 .

- The independent theory gives good predictions for the bulk behavior of highly porous systems $(\varepsilon \geqslant 0.992)$ for all cases considered. However, because the bulk properties no longer hold good near the bounding surface, the boundary behavior predicted by the independent theory is different from that predicted by the Monte Carlo simulations. As a result, the transmittance predicted by the independent theory

Tablc 1. Radiation exchange factor $F(s=0.4)$

\begin{tabular}{lccccc}
\multicolumn{1}{c}{ Model } & 0.2 & 0.35 & 0.60 & 0.85 & 10 \\
\hline Two-flux (diffuse) & 0.88 & 0.91 & 1.02 & 1.06 & 1.11 \\
Two-flux (specular) & 1.11 & 1.11 & 1.11 & 1.11 & 1.11 \\
Discrete ordinates (diffuse) & 1.09 & 1.15 & 1.25 & 1.38 & 1.48 \\
Discrete ordinates (specular) & 1.48 & 1.48 & 1.48 & 1.48 & 1.48 \\
Argo and Smith & 0.11 & 0.21 & 0.43 & 0.74 & 1.0 \\
Vortmeyer & 0.25 & 0.33 & 0.54 & 0.85 & 1.12 \\
Kasparek (experiment) & - & 0.54 & -- & 1.02 & - \\
Monte Carlo (diffuse) & 0.32 & 0.45 & 0.68 & 0.94 & 1.10 \\
Monte Carlo (specular) & 0.34 & 0.47 & 0.69 & 0.95 & 1.10 \\
\hline
\end{tabular}


(for $\varepsilon=0.992$ ) is always slightly more than that predicted by the Monte Carlo method.

- Two distinct dependent scattering effects were identified. The multiple scattering of the reflected rays increases the effective scattering and absorption cross sections of the particles. This results in a decrease in transmission through the bed. The transmission through a particle in a packed bed results in a decrease in the effective cross sections resulting in an increase in the transmission through a bed. For opaque particles, only the multiple scattering effect is found while for transparent and semi-transparent particles, both the above-mentioned effects are found and tend to oppose each other.

- Fair agreement with the Chen and Churchill experiment on steel spheres is found using the Monte Carlo method. More importantly, the inability of the independent theory to match the experimental results is clearly demonstrated. The Monte Carlo results show good agreement with the Chen and Churchill experiment with glass spheres. However, the deviation from the independent theory is not large, because of the low absorption of the glass spheres.

- The agreement of the Monte Carlo results with the Kasparek experiment is encouraging. The independent theory fails to give acceptable results for a packed bed of absorbing, emitting, and scattering spheres.

- The two-flux model fails for absorbing media, even when the phase function is isotropic. The transmission through such media was found to be severely underpredicted by the two-flux model.

Acknowledgement--We are grateful to Professor E. M. Tory for the use of his computer program on random packing of spherical particles.

\section{REFERENCES}

1. R. Siegel and J. R. Howell, Thermal Radiation Heat Transfer, 2nd Edn. McGraw-Hill, New York (1981).

2. M. N. Ozisik, Radiative Transfer and Interaction with Conduction and Convection. Werbel \& Peck, New York (1985).

3. H. C. Hottel, A. F. Sarofim, W. H. Dalzell and I. A. Vasalos, Optical properties of coatings, effect of pigment concentration, AIAA J. 9, 1895-1898 (1971).

4. M. Q. Brewster, Radiative heat transfer in fluidized bed combustors, ASME Paper No. 83-WA/HT-82 (1983).

5. Y. Yamada, J. D. Cartigny and C. L. Tien, Radiative transfer with dependent scattering by particles : Part 2experimental investigation, J. Heat Transfer 108, 614 618 (1986).

6. B. L. Drolen and C. L. Tien, Independent and dependent scattering in packed spheres systems, $J$. Thermophys. Heat Transfer 1, 63-68 (1987).

7. A. Ishimaru and Y. Kuga, Attenuation constant of a coherent field in a dense distribution of particles, J. Opt. Soc. Am. 72, 1317-1320 (1982).

8. J. D. Cartigny, Y. Yamada and C. L. Tien, Radiative heat transfer with dependent scattering by particles : Part 1 -theoretical investigation. J. Heat Transfer 108, 608613 (1986).

9. S. Kumar and C. L. Tien, Dependent scattering and absorption of radiation by small particles, J. Heat Transfer 112, 178-185 (1990).

10. Y. S. Yang, J. R. Howell and D. E. Klein, Radiative heat transfer through a randomly packed bed of spheres by the Monte Carlo method, J. Heat Transfer 105, 325332 (1983).

11. K. Kudo, W. J. Yang, H. Tanaguchi and H. Hayasaka, Radiative heat transfer in packed spheres by Monte Carlo method. In Heat Transfer in High Technology and Power Engineering Proceedings, pp. 529-540. Hemisphere, New York (1987).

12. C. L. Tien and B. L. Drolen, Thermal radiation in particulate media with dependent and independent scattering, A. Rev. Numer. Fluid Mech. Heat Transfer 1, 132 (1987).

13. J. C. Chen and S. W. Churchill, Radiant heat transfer in packed beds, A.I.Ch.E. Jl9, 35-41 (1963).

14. D. Vortmeyer, Radiation in packed solids, Proc 6th Int. Heat Transfer Conf., Vol. 6, pp. 525-539 (1978).

15. M. Kaviany, Principles of heat transfer in porous media, monograph. Springer, Berlin (in press).

16. B. G. Carlson and K. D. Lathrop, Transport theorythe method of discrete ordinates. In Computing Methods in Reactor Physics (Edited by H. Greenspan, C. N. Kelber and D. Okrent), pp. 171-266. Gordon \& Breach Science, New York (1968).

17. W. A. Fiveland, Discrete ordinate methods for radiative heat transfer in isotropically and anisotropically scattering media, J. Heat Transfer 109, 809-812 (1987).

18. S. Kumar, A. Majumdar and C. L. Tien, The differentialdiscrete ordinate method for solution of the equation of radiative transfer, J. Heat Transfer 112, 424-429 (1990).

19. C. K. Chan and C. L. Tien, Radiative transfer in packed spheres, J. Heat Transfer 96, 52-58 (1974).

20. W. S. Jodrey and E. M. Tory, Simulation of random packing of spheres, Simulation Jan., 1-12 (1979).

21. H. C. van de Hulst, Light Scattering by Small Particle. Dover, New York (1981).

22. M. Q. Brewster and C. L. Tien, Examination of the twoflux model for radiative transfer in particulate systems, Int. J. Heat Mass Transfer 25, 1905-1907 (1982).

23. M.P. Mengüc and R. Viskanta, Comparison of radiative heat transfer approximations for highly forward scattering planar medium, ASME Paper No. 82-HT-20 (1982).

24. J. C. Chen, Radiant heat transfer in packed media, Ph.D. Thesis, University of Michigan, Ann Arbor, Michigan (1961).

25. R. C. Weast, Handbook of Chemistry and Physics, 68th Edn. CRC Press, Boca Raton, Florida (1987).

26. C. K. Hsieh and K. C. Su, Thermal radiative properties of glass from 0.32 to $206 \mu \mathrm{m}$, Solar Energy 22, 37-43 (1979). 


\title{
THEORIE INDEPENDANTE CONTRE SIMULATION DIRECTE DU TRANSFERT THERMIQUE RADIATIF DANS LES LITS FIXES
}

Résumé-On considère le rayonnement thermique dans les lits fixes de grosses particules sphériques. Lat pratique courante est de suivre la théorie de la dispersion indépendante tant que $C, \lambda>0,5$ ou $C$ est la distance libre moyenne entre particules et $;$ la longueur d'onde. Les propriétés de la particule seule sont reliées aux propriétés radiatives du lit par une moyenne en volume. Léquation de transfert est ensuite résolue par une méthode approchée telle que la méthode des ordonnées discrètes ou celle des deux flux. Dans cette étude, on utilise la méthode de Monte Carlo pour examiner le transfert radiatif a travers les lits fixes de grosses (domaine géométrique) particules. On considère des particules opaques, semi-transparentes et émissives. Les résultats sont comparés à la théorie indépendante el aux résultats expérimentaux disponibles; ils indiquent que la théorie indépendante lombe en défaut quand ce critère $C$ : est satisfait. Le succès de la théoric indépendante dans les systemes à faible porosité, noté par des chercheurs antérieurs, est montré être soit un cas spécial existant seulement pour un petit domaine de propriétés optiques, soit dủ à des hypotheses injustifiées. Pour les mômes propriétés radiatives de particule, la déviation par rapport à la théorie indépendante augmente quand la porosité décroit. Cette déviation peut êtro sensible même pour des porosités allant jusqu'ì 0.935 .

\section{WÄRMETRANSPORT DURCH STRAHLUNG IN SCHÜTTUNGEN: VERGLEICH DER UNABHÄNGIGEN THEORIE MIT EINER DIREKTEN SIMULATION}

\begin{abstract}
Zusammenfassung - Es wird der Wärmetransport durch Strahlung in Schüttungen mit verhältnismäßig großen kugeligen Partikeln betrachtet. Üblicherweise wird dazu die Theorie der unabhängigen Streuung verwendet, solange $C / \gamma_{>}>0,5$ ist (mit $C$ und $\lambda$ als der mittleren freien Weglänge bzw. der Wellentänge). Die Eigenschaften des einzelnen Partikels werden durch Volumenmittelung zu Strahlungseigenschaften für die Schüttung verarbeitet. Die Transportgleichung wird mit Hilfe eines Näherungsverfahrens gelöst. beispielsweise mit der Methode diskreter Ordinaten oder mit der Zwei-Strom-Methode. In der vorliegenden Untersuchung wird die Monte-Carlo-Methode verwendet, um den Wärmetransport durch Strahlung in Schüttungen aus großen Partikeln zu bestimmen. Es werden strahlungsundurchlässige, halbdurchlässige und emittierende Partikel untersucht. Die Ergebnisse werden mit solchen nach der unabhängigen Theorie und mit verfügbaren Versuchsdaten verglichen. Es zeigt sich. daß die unabhängige Theorie falsche Ergebnisse liefert, sogar dann, wenn das C angemerkte Anwendbarkeit der unabhängigen Theorie bei Systemen mit geringer Porosität sich entweder auf Spezialfälle beschränkt, die nur für cinen engen Bereich der optischen Eigenschaften existieren, oder aber durch nichtzulässige Annahmen gewonnen wird. Bei identischen Strahlungseigenschaften der Partikel nimmt die Abweichung von der unabhängigen Theorie mit abnehmender Porosität zu. Diese Abweichung kann signifikant sein- - selbst für Porositäten um 0.935.
\end{abstract}

\section{СРАВНЕНИЕ ТЕОРИИ ПІЕЗАВИСИМОГО РАССЕЯНИЯ И ПРЯМОГО МОДЕЛИРОВАНИЯ РАДИАЦИОННОГО ТЕПЛОПЕРЕНОСА В УПАКОВАННЫХ СЛОЯХ}

\begin{abstract}
Аннотаци-Исследуется радиационный теплоперенос в упакованных слоях относительно крупных сферических частиц. Общепринятым является использование теории независимого рассеяния при $C / \lambda>0,5$, где $C$-среднее расстояние между частицами, $\lambda$-длина волны. Методом усреднения по объему устанавливается взаимосвязь между свойствами единичной частицы и излучательными свойствами слоя. Затем одним из приближенных методов-методом дискретиых ординат или двухпоточным--решается уравнение переноса. В данной работе для исследования радиационног тенлопереноса через упакованные слои крупных частиц (геомстричсскос приближение) используется метод Монте Карло. Рассматриваются непрозрачные, полупрозрачные и излучающие частицы. Сравнение полученных результатов с теорией независимого рассеяния и имеюцимися экспериментальными ланными показывает, что упомянутая теория непригодна даже при выполнении критерия для $C / \lambda$. Как отмечено в предыдущих исследованиях, применение такой теории для систем с низкой порозностью является успешным только в отдельных случаях в узком диапазоне оптических свойств или при наличии некоторых неправомерных допущений. Показано, что при одинаковых излучательных свойствах частиц отклонение от резу льтатов теории независкммого рассеяния возрастает с уменъшением прозности. Указанное отклонение может быть существенным даже в случае значения порозности, равного 0,935 .
\end{abstract}

